\title{
Cochrane Review Summary: Pelvic floor muscle training added to another active treatment versus the same active treatment alone for urinary incontinence in women
}

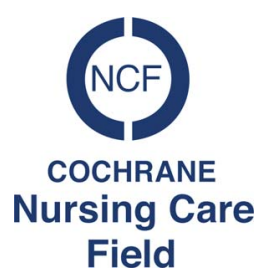

\section{Daksha Trivedi}

Senior Research Fellow, Evidence Based Practice, Centre for Research in Primary and Community Care, University of Hertfordshire, Hatfield, UK

Keywords: pelvic floor muscle training; urinary incontinence; women

First published online 5 August 2015

\section{Review question}

What are the effects of pelvic floor muscle training (PFMT) given in combination with other treatments to women with urinary incontinence?

\section{Relevance to primary care and nursing}

Urinary incontinence is a common problem in women who report a wide range of urinary symptoms, which progress with age and significantly affect the overall quality of life (QoL). Estimated prevalence rates range from 30 to $60 \%$ in middleaged and older women and rise with advancing age. Although a number of conservative, pharmacological and surgical treatment options are available, the financial impact of the condition is substantial. Primary health-care nurses and doctors have a key role in the management of urinary incontinence in women (National Institute for Health and Clinical Excellence, 2013) and this systematic review aimed to determine the effectiveness of adding PFMT to any of the other active treatments.

Correspondence to: Dr Daksha Trivedi, Senior Research Fellow, Evidence Based Practice, Centre for Research in Primary and Community Care, University of Hertfordshire, College Lane, Hatfield, AL10 9AB, UK. Email: d.trivedi@herts.ac.uk

\section{Characteristics of the evidence}

This Cochrane review contained 11 randomised controlled trials targeting adult women with clinical or urodynamic evidence of stress urinary incontinence (SUI), urgency urinary incontinence (UUI) or mixed urinary incontinence (MUI) (Ayeleke et al., 2013).

Included trials compared PFMT added to another active treatment $(n=494)$ with the same active treatment alone $(n=490)$. PFMT was defined as a programme of repeated voluntary pelvic floor muscle contractions taught and/or supervised by health-care professionals. PFMT programmes varied considerably; common variations included number and frequency of contractions, use of biofeedback and number of health professional contacts. PFME programmes were also commonly accompanied by advice on managing urgency and frequency, and lifestyles advice (e.g., weight reduction). PFMT programmes ranged from 4 to 12 weeks long, and addressed muscle strength, endurance, coordination or a combination of these (although in six trials insufficient description made the exercise difficult to characterise). Active comparators included vaginal cones, bladder training, electrical stimulation, continence pessary, various drug therapies and others such as heat and steam generating sheets. The interventions were delivered by nurses, physical therapists and/or doctors. 
Exclusions: studies of antenatal or postnatal women, or women whose symptoms were complicated by other co-morbidities (e.g., cognitive impairment, neurological conditions, frailty).

\section{Summary of key evidence}

Most included trials were assessed as unclear risk of bias for most of the methodological domains, and the overall quality of evidence was rated as low. Moreover, most of the included trials did not report outcomes that were important from patients' perspective. Meta-analysis was not possible as most comparisons were reported by single trials and outcomes. The total number of participants and effect sizes, means and risk ratio (RR), 95\% confidence intervals (CI) are shown in parentheses where appropriate. The findings are summarised according to types of interventions.

Primary outcomes: number of women reporting 'cured' (100\% reduction) or 'improved' $(\geqslant 50 \%$ reduction) in incontinence; condition-specific QoL; patient global impression of improvement.

Secondary outcomes: frequency of incontinence episodes; objective assessment of improvement; number of continence pads used; frequency of micturition; volume of urine per micturition.

Other outcomes: patient satisfaction with treatment outcome.

\section{Physical interventions}

PFMT added to vaginal cones versus vaginal cones alone: one trial of women with SUI $(n=42)$ showed no significant effect on women cured or improved (objective assessment) with SUI at 12 weeks.

\section{Behavioural interventions}

PFMT added to bladder training versus bladder training alone in women with SUI, UUI or MUI: one trial $(n=135)$, reported a cure or an improvement in incontinence and QoL immediately after treatment, but not at three months. There was a non-significant reduction in the frequency of incontinence episodes, and no differences in the number of women who relapsed from three months to three years follow-up.

Primary Health Care Research \& Development 2015; 16: 545-547

\section{Electrical/magnetic interventions}

PFMT added to electrical stimulation versus electrical stimulation alone (excluding implanted electrodes): one small trial $(n=22)$ showed no significant effect of the intervention on cure or improvement in women with SUI at six weeks. Effect size in one small study examining magnetic stimulation was not estimable.

\section{Mechanical interventions}

PFMT added to continence pessaries versus continence pessaries alone: evidence from one trial $(n=299)$ showed no significant effect on cure or improvement, QoL or on patient global impression of improvement or patient satisfaction at 3,6 or 12 months in women with SUI.

\section{Drug interventions with and without PFMT}

Each drug was tested only in single trials. PFMT added to duloxetine $(n=104)$, oxybutynin $(n=64)$, solifenacin $(n=162)$ and clenbutarol $(n=41)$ showed no significant effect on any reported outcomes. Only one small trial $(n=29)$ testing an unspecified drug therapy showed that significantly more women reported benefit from combined treatment (RR 2.57, 95\% CI 1.06-6.20).

\section{Other interventions}

PFMT plus heat and steam generating sheet versus heat and steam generating sheet alone: only one trial $(n=74)$ of women with UUI or MUI showed a significant effect of the combined intervention on number of women cured (RR 2.38, 95\% CI 1.19-4.73).

\section{Implications for practice}

Most evidence came from small under-powered trials, making it difficult to draw conclusions from insufficient evidence on effectiveness or efficacy of PFMT, as most included trials did not examine adverse events associated with PFMT.

\section{Implications for research}

The overall quality of evidence was low and highquality research is required to evaluate the effect of adjunctive PFMT for urinary incontinence in women. None of the trials investigated PFMT with 
and without surgical intervention or lifestyle interventions, which warrant further investigations. There is a need to focus on QoL, socioeconomic implications, long-term effects and adverse events associated with combining PFMT with other active treatments.

\section{Acknowledgement}

The author is a member of the Cochrane Nursing Care Field (CNCF).

\section{Financial Support}

This research received no specific grant from any funding agency, commercial or not-for-profit sectors.

\section{Conflicts of Interest}

None.

\section{References}

Ayeleke, R.O., Hay-Smith, E.J.C. and Omar, M.I. 2013: Pelvic floor muscle training added to another active treatment versus the same active treatment alone for urinary incontinence in women. Cochrane Database of Systematic Reviews, Issue 11, Art. No.: CD010551. doi: 10.1002/ 14651858.CD010551.pub2.

National Institute for Health and Clinical Excellence. 2013: Urinary incontinence: the management of urinary incontinence in women, NICE. Retrieved 26 May 2015 from https://www.nice.org.uk/guidance/cg171 